\title{
ANÁLISE DO DESENVOLVIMENTO NEUROPSICOMOTOR DE CRIANÇAS PARTICIPANTES DE UM PROGRAMA MÃE-BEBÊ
}

\author{
Analysis of neuropsychomotor development of children participating in a mother- \\ baby program
}

\author{
Análisis del desarrollo neuropsicomotor de niños participantes de un programa \\ madre-bebé
}

\author{
Simone de Paula (iD \\ Universidade Feevale - FEEVALE - Novo Hamburgo (RS) - Brasil \\ Ellen Becker Rohr (iD \\ Universidade Feevale - FEEVALE - Novo Hamburgo (RS) - Brasil \\ Maristela Cassia de Oliveira Peixoto (iD \\ Universidade Feevale - FEEVALE - Novo Hamburgo (RS) - Brasil \\ Caroline D'Azevedo Sica iD \\ Universidade Feevale - FEEVALE - Novo Hamburgo (RS) - Brasil \\ Ilse Maria Kunzler (DD \\ Universidade Feevale - FEEVALE - Novo Hamburgo (RS) - Brasil
}

\section{RESUMO}

Objetivo: Analisar o desenvolvimento neuropsicomotor de crianças participantes de um Programa Mãe-bebê. Métodos: Estudo transversal, realizado com 52 crianças entre 6 e 12 meses de idade, procedentes do Programa Mãe-bebê, um projeto de extensão realizado em uma comunidade carente de Novo Hamburgo, Rio Grande do Sul, Brasil, em 2017. A partir dos prontuários, selecionaram-se as crianças e, para a avaliação do desenvolvimento infantil, utilizou-se o Teste de Triagem de Desenvolvimento de Denver II, aplicado no domicílio das crianças, por profissionais treinados, no período de março a novembro de 2017. Resultados: A amostra compôs-se de crianças nascidas a termo (38,52 $\pm 1,83$ semanas), com peso acima de $2500 \mathrm{~g}(94,23 \%$; $\mathrm{n}=49)$, que receberam aleitamento materno exclusivo $(57,70 \% ; n=30)$. Quanto ao desenvolvimento neuropsicomotor, $30,8 \%(n=16)$ das crianças apresentam desenvolvimento global suspeito, sendo que os domínios mais afetados foram a linguagem $(13,5 \%$; $n=7)$ e o motor grosso $(11,5 \% ; n=6)$. As variáveis independentes que mostraram associação com suspeita de atraso no desenvolvimento neuropsicomotor foram o aleitamento materno exclusivo $(p=0,03)$, o número de filhos $(p=0,01)$ e a situação conjugal $(p=0,04)$. Conclusão: A maioria das crianças $(69,2 \%, n=36)$ participantes do Programa Mãe-bebê apresentaram um desenvolvimento neuropsicomotor adequado e compatível com sua faixa etária. No entanto, $30 \%(n=16)$ das crianças que apresentaram um neurodesenvolvimento considerado suspeito tiveram a linguagem e o motor grosso como os domínios mais afetados.

Descritores: Desenvolvimento Infantil; Promoção da Saúde; Atenção Primária à Saúde.

\section{ABSTRACT}

Objective: To analyze the neuropsychomotor development of children participating in a Mother-baby Program. Methods: A crosssectional study was carried out with 52 children between 6 and 12 months of age, from the Mother-baby Program, an extension project carried out in a deprived community of Novo Hamburgo, Rio Grande do Sul, Brazil, in 2017. The children were selected from the records and to evaluate child development, the Denver II Development Screening Test was applied in the children's home by trained professionals during the period from March to November 2017. Results: The sample consisted of infants born at term (38.52 \pm 1.83 weeks), weighing more than $2500 \mathrm{~g}(94.23 \% ; n=49)$, who received exclusive breastfeeding $(57.70 \% ; n=$ 30). As to neuropsychomotor development, $30.8 \%(n=16)$ of the children presented suspicious global development, with the most affected domains being language $(13.5 \% ; n=7)$ and gross motor $(11.5 \% ; n=6)$. The independent variables that showed an association with suspected delay in neuropsychomotor development were exclusive breastfeeding $(p=0.03)$, number of children 
( $p=0.01)$ and marital status $(p=0.04)$. Conclusion: The majority of the children $(69.2 \%, n=36)$ participants in the Mother-baby Program presented adequate neuropsychomotor development compatible with their age group. However, $30 \%(n=16)$ of the children who had neurodevelopment considered suspect had the language and the motor gross as the most affected domains.

Descriptors: Child Development; Health Promotion; Primary Health Care.

\section{RESUMEN}

Objetivo: Analizar el desarrollo neuropsicomotor de niños participantes de un Programa Madre-bebé. Métodos: Estudio transversal realizado con 52 niños entre los 6 y 12 meses de edad de un Programa Madre-bebé que es un proyecto de extensión realizado en una comunidad que está falta de condiciones socio-económicas de Novo Hamburgo, Rio Grande de Sur, Brasil, en 2017. Se ha elegido los niños a partir de los historiales clínicos y se ha aplicado la Prueba de Tamizaje del Desarrollo de Denver Il para la evaluación del desarrollo infantil en los domicilios de los niños por profesionales entrenados en el periodo entre marzo y noviembre de 2017. Resultados: La muestra ha sido de niños nacidos a término $(38,52 \pm 1,83$ semanas), con más de $2500 \mathrm{~g}$ de peso $(94,23 \% ; n=49)$ y que han recibido amamantamiento materno exclusivo $(57,70 \% ; n=30)$. Respecto el desarrollo neuropsicomotor el 30,8\% $(n=16)$ de los niños presentan el desarrollo global sospechoso con los dominios de lenguaje (13,5\%; $n=7)$ y motor grueso $(11,5 \% ; n=6)$ más afectados. Las variables independientes que señalaron asociación sospechosa para el retraso del desarrollo neuropsicomotor fueron el amamantamiento materno exclusivo $(p=0,03)$, el número de hijos ( $p=0,01)$ y la situación conyugal $(p=0,04)$. Conclusión: La mayoría de los niños $(69,2 \%, n=36)$ participantes del Programa Madre-bebé presentaron un desarrollo neuropsicomotor adecuado y compatible con su franja de edad. Sin embargo, el 30\% ( $n=16$ ) de los niños que presentaron un desarrollo neurológico sospechoso tuvieron los dominios de lenguaje y motor grueso más afectados.

Descriptores: Desarrollo Infantil; Promoción de la Salud; Atención Primaria de Salud.

\section{INTRODUÇÃO}

O desenvolvimento neuropsicomotor é um processo único de cada criança que tem como finalidade sua inserção na sociedade em que vive. Em virtude da intensa capacidade que o sistema nervoso possui de reorganizar e adaptar suas redes neuronais em resposta às exigências ambientais e orgânicas, o período da infância é expresso por avanços significativos nas habilidades motoras, cognitivas, psicossociais e de linguagem, com aquisições progressivamente mais complexas nas funções da vida diária. O período pré-natal e os primeiros anos de vida são o alicerce desse processo, que decorre da interação de características biopsicológicas, herdadas geneticamente, e experiências oferecidas pelo meio ambiente. As experiências são constituídas pelo cuidado que a criança recebe e pelas oportunidades que ela tem para exercitar ativamente suas habilidades ${ }^{(1-3)}$.

$O$ cuidado voltado às necessidades de desenvolvimento possibilita à criança alcançar todo o seu potencial em cada fase, com repercussões positivas na idade adulta(2). No entanto, estima-se que mais de 250 milhões de crianças com menos de 5 anos estão em risco para atraso nas aquisições neuropsicomotoras, podendo resultar, em longo prazo, em prejuízos de aprendizagem e déficit aproximado de $20 \%$ da renda anual na vida adulta ${ }^{(4)}$.

Nesse contexto, o Ministério da Saúde determina que o acompanhamento do desenvolvimento da criança na atenção básica objetiva sua promoção, proteção e a deteç̧ão precoce de alterações passíveis de modificação que possam repercutir em sua vida futura. Isto ocorre, principalmente, por meio de ações educativas e de acompanhamento integral da saúde da criança( ${ }^{(5)}$. Adicionalmente, a Política Nacional de Promoção da Saúde acrescenta que os procedimentos de acompanhamento do desenvolvimento infantil nos seus aspectos motor, cognitivo e emocional devem ser valorizados nos programas de saúde da criança como uma importante estratégia de prevenção de deficiências nessa população( ${ }^{(6)}$.

Desse modo, para a plena promoção da saúde da criança é indispensável a compreensão sobre os aspectos multifatoriais associados ao desenvolvimento infantil, incluindo não somente fatores biológicos, mas também a influência de requisitos psicossociais que afetam adversamente a aquisição de marcos do desenvolvimento ${ }^{(7)}$. Dentre os fatores de risco para atrasos no desenvolvimento, destaca-se a inadequada condição nutricional da criança, o baixo nível de estimulação no ambiente familiar e as fragilidades culturais e sociodemográficas da família. Todos esses aspectos, associados com a pobreza, podem levar à desigualdades no desenvolvimento precoce da criança e, consequentemente, prejudicar seu nível educacional e sua produtividade na vida adulta, perpetuando, dessa forma, o ciclo da pobreza ${ }^{(4)}$.

Em consonância com a Política Nacional de Atenção Integral à Saúde da Criança (PNAISC) $)^{(8)}$, o Programa de Extensão Comunitária Mãe-Bebê, da Universidade Feevale, surgiu em 2016 com o objetivo de atuar na promoção 
da saúde da mulher no ciclo gravídico-puerperal, do neonato e da criança até 1 ano através de ações integradas e interdisciplinares de atenção à saúde, com enfoque na atenção ao parto e ao nascimento e em sua repercussão sobre a mortalidade neonatal e materna. Nesse programa, para a vigilância do desenvolvimento infantil e de indicadores de saúde materno-infantil, as famílias participantes recebem assistência mensal de acadêmicos e profissionais das áreas de Nutrição, Fisioterapia, Enfermagem e Psicologia, através de visitas domiciliares. Em virtude da importância de desenvolver estratégias de promoção de saúde voltadas para o desenvolvimento da criança e para a redução da morbimortalidade infantil, o objetivo do presente estudo foi analisar o desenvolvimento neuropsicomotor de crianças participantes de um Programa Mãe-bebê.

\section{MÉTODOS}

Estudo transversal, observacional e descritivo, realizado com 52 crianças entre 6 e 12 meses de idade, de ambos os sexos, participantes do Programa de Extensão Comunitária Mãe-bebê da Universidade Feevale, Rio Grande do Sul, Brasil. O programa é desenvolvido em um bairro da periferia da cidade de Novo Hamburgo, que possui em torno de 10.981 pessoas adscritas em quatro Estratégias de Saúde da Família. Além dos elevados índices de violência e do prevalente envolvimento com o tráfico de drogas, o perfil sociodemográfico dessa comunidade é caracterizado por pessoas com baixa renda e escolaridade, e moradores de áreas de invasão, com saneamento básico precário - características que fazem com que as famílias residentes estejam em situação de vulnerabilidade social.

Atuando de forma interdisciplinar, o Programa Mãe-bebê realiza o acompanhamento individual das famílias beneficiadas através de visitas domiciliares e entrevistas em consultórios da Unidade de Saúde da Família, atuando na promoção da saúde da criança até os 12 meses de vida. Além da avaliação do crescimento e desenvolvimento do neonato e do lactente, as famílias recebem orientações sobre o calendário vacinal, os cuidados adequados com o bebê, a importância do aleitamento materno exclusivo, o fortalecimento de vínculos familiares, entre outros. As atividades ocorrem três vezes por semana, com a participação de todas as áreas da saúde e em alinhamento com a equipe de saúde da unidade.

Neste estudo, excluíram-se as crianças com diagnóstico de desordens neurológicas ou que estivessem pouco colaborativas no momento do teste. Somente as crianças cujos pais e/ou responsáveis aceitaram o convite para participar e assinaram o Termo de Consentimento Livre e Esclarecido foram incluídas na pesquisa.

Realizou-se a seleção das crianças a partir da análise de prontuários do Programa Mãe-bebê, que continham informações clínicas, gestacionais e sociodemográficas sobre as famílias acompanhadas. Após a seleção dos participantes da pesquisa, realizou-se a avaliação do desenvolvimento infantil através do Teste de Triagem de Desenvolvimento de Denver II (TTDDII), seguindo as instruções do manual do teste ${ }^{(9)}$. O teste é composto de 125 itens distribuídos em 4 domínios do desenvolvimento: pessoal-social, motor fino-adaptativo, linguagem e motor grosso. Após a identificação da faixa etária da criança, traçou-se uma linha vertical correspondente à idade. Avaliaram-se todos os itens cortados pela linha, bem como os três itens totalmente à esquerda da linha em cada área de desenvolvimento, interpretando-se como: $(\mathrm{N})$ normal, quando a criança realiza um item cortado pela linha; (C) cautela, quando a criança falha em um item no qual a linha da idade cruza entre 75 a $90 \%$; e (A) atraso, quando a criança falha em um item que está totalmente à esquerda da linha da idade. Após interpretar cada item, o escore final do teste determina duas classificações: desenvolvimento adequado, quando não há atrasos ou no máximo 1 cautela; e desenvolvimento suspeito, quando há 2 ou mais cautelas e/ou 1 ou mais atrasos.

Realizaram-se as avaliações do desenvolvimento no ano de 2017, nos meses de março a novembro, sendo conduzidas por profissionais da saúde submetidos à prévio treinamento para a utilização do teste. A avaliação das crianças ocorreu individualmente, em seu domicílio, e os materiais utilizados formavam um kit padrão contendo blocos de madeira quadrados e coloridos, uvas passas, boneca de plástico, canecas e bola. As mães ou responsáveis pela criança participaram e auxiliaram no relato de algumas atividades previstas no teste. O tempo total para a aplicação do teste foi de, aproximadamente, 30 minutos.

Realizou-se a análise quantitativa dos dados por meio de estatística descritiva, sendo os resultados apresentados em números absolutos e percentuais, sob a forma de tabelas, considerando-se os critérios de interpretação dos resultados do teste. Também se estudou a associação entre a variável dependente e as independentes, utilizando-se o teste de Qui-quadrado e os valores de odds ratio com seus respectivos intervalos de confiança de $95 \%$. Para obter os valores de odds ratio, empregou-se o modelo de regressão logística (método backward stepwise), usandose o software SPSS 11.0. Consideraram-se significantes as variáveis com $p<0,05$. Para o cálculo do tamanho da amostra, levou-se em consideração a prevalência de $33 \%$ de atraso no desenvolvimento neuropsicomotor ${ }^{(10)}$, com 
intervalo de $90 \%$ de confiança, erro amostral de $5 \%$ e uma população de 61 crianças atendidas no Programa Mãebebê. Com base nesses parâmetros, considerou-se o tamanho amostral mínimo de 59 crianças.

Este estudo foi aprovado pelo Comitê de Ética em Pesquisa da Universidade Feevale sob o Parecer n. ${ }^{\circ}$ 2.340.689 e está de acordo com as normas vigentes na Resolução n. ${ }^{\circ}$ 466/12 do Conselho Nacional de Saúde, e suas complementares, que regulamentam a pesquisa envolvendo seres humanos ${ }^{(11)}$.

\section{RESULTADOS}

Pode-se observar que a amostra foi composta por 39 bebês do sexo masculino e 13 do sexo feminino, predominantemente nascidos a termo e com peso ao nascimento acima de $2500 \mathrm{~g}$, avaliadas através da TTDDII no terceiro trimestre de vida. A maioria nasceu de parto vaginal $(67,30 \% ; n=35)$ e recebeu leite materno, pelo menos, até os seis meses de idade $(57,70 \% ; n=30)$. Em relação às questões socioeconômicas, observa-se que as famílias das crianças avaliadas vivem com mais de 1 salário mínimo mensal e que as mães, em sua maioria, possuem menos de 2 filhos e contam com a presença de um companheiro, apesar da baixa escolaridade. As principais características das mães e das crianças participantes deste estudo estão descritas na Tabela I.

Tabela I - Características das mães e das crianças participantes do estudo $(n=52)$. Novo Hamburgo, Rio Grande do Sul, 2017.

\begin{tabular}{|c|c|}
\hline Característica & Média \pm DP \\
\hline Idade da mãe (anos) & $26,71 \pm 6,20$ \\
\hline Idade da criança na avaliação (meses) & $8,65 \pm 3,01$ \\
\hline Número de filhos & $1,69 \pm 1,00$ \\
\hline Idade gestacional ao nascimento (semanas) & $38,52 \pm 1,83$ \\
\hline Peso ao nascimento $(\mathrm{g})$ & $3290,50 \pm 537,34$ \\
\hline Característica & $\%(n)$ \\
\hline \multicolumn{2}{|l|}{ Peso ao nascimento } \\
\hline$\geq 2500 \mathrm{~g}$ & $94,23(49)$ \\
\hline$<2500 \mathrm{~g}$ & $5,76(3)$ \\
\hline \multicolumn{2}{|l|}{ AME } \\
\hline Sim & $57,70(30)$ \\
\hline Não & $42,30(22)$ \\
\hline \multicolumn{2}{|l|}{ Tipo de parto } \\
\hline Vaginal & $67,30(35)$ \\
\hline Cesariana & $32,69(17)$ \\
\hline \multicolumn{2}{|l|}{ Renda familiar mensal } \\
\hline$<1$ salário mínimo & $32,69(17)$ \\
\hline$\geq 1$ salário mínimo & $67,30(35)$ \\
\hline \multicolumn{2}{|l|}{ Idade materna } \\
\hline$<18$ anos & $3,85(2)$ \\
\hline$\geq 18$ anos & $96,15(50)$ \\
\hline \multicolumn{2}{|l|}{ Escolaridade materna } \\
\hline$\leq 8$ anos & $67,30(35)$ \\
\hline$>8$ anos & $32,69(17)$ \\
\hline \multicolumn{2}{|l|}{ Número de filhos } \\
\hline$\leq 2$ & $86,53(45)$ \\
\hline$>2$ & $13,46(7)$ \\
\hline \multicolumn{2}{|l|}{ Situação conjugal } \\
\hline Vive com companheiro & $80,76(42)$ \\
\hline Vive sem companheiro & $19,23(10)$ \\
\hline
\end{tabular}

AME: aleitamento materno exclusivo até os 6 meses de vida; \%: porcentagem; n: número absoluto

Quanto às variáveis relacionadas ao desenvolvimento neuropsicomotor, 30,8\% das crianças apresentaram desenvolvimento global suspeito, segundo o TTDDII (Tabela II), sendo que os domínios mais afetados foram a linguagem e o motor grosso, com percentuais de alterações no desenvolvimento de $13,5 \%$ e $11,5 \%$, respectivamente. 
Tabela II - Desempenho global e por domínio das crianças no Teste de Triagem de Desenvolvimento de Denver II $(n=52)$. Novo Hamburgo, Rio Grande do Sul, 2017.

\begin{tabular}{lc}
\hline Escore global & $\%$ (n) \\
\hline Desenvolvimento adequado & $69,2(36)$ \\
Desenvolvimento suspeito & $30,8(16)$ \\
\hline Domínios & \\
\hline Pessoal-social & $94,2(49)$ \\
Desenvolvimento adequado & $5,8(3)$ \\
Desenvolvimento suspeito & \\
Motor Fino-adaptativo & $94,2(49)$ \\
Desenvolvimento adequado & $5,8(3)$ \\
Desenvolvimento suspeito & \\
Linguagem & $86,5(13,5)$ \\
Desenvolvimento adequado & $13,5(7)$ \\
Desenvolvimento suspeito & \\
Motor Grosso & $88,5(46)$ \\
Desenvolvimento adequado & $11,5(6)$ \\
Desenvolvimento suspeito & \\
\hline
\end{tabular}

$\%$ : porcentagem; n: número absoluto

A Tabela III mostra os resultados referentes à regressão logística e ao percentual de desenvolvimento suspeito em cada uma das variáveis analisadas. As variáveis independentes que mostraram associação significativa com suspeita de atraso no desenvolvimento neuropsicomotor foram: o aleitamento materno exclusivo, sendo que crianças que não foram amamentadas tiveram 6,93 vezes mais chances de apresentar suspeita no atraso no desenvolvimento neuropsicomotor quando comparadas a crianças que foram amamentadas até os 6 meses; o número de filhos, que aumentou em 16,94 vezes a chance de apresentar um desenvolvimento suspeito em crianças procedentes de famílias com 3 filhos ou mais; e a situação conjugal, que indicou que as crianças que vivem em famílias sem a presença do companheiro tiveram 8,31 vezes mais chances de apresentar suspeita para atraso no desenvolvimento neuropsicomotor.

Tabela III - Análise entre variáveis independentes e o desfecho suspeita de atraso no desenvolvimento neuropsicomotor, segundo o Teste de Triagem de Desenvolvimento de Denver II. Novo Hamburgo, Rio Grande do Sul, 2017.

\begin{tabular}{|c|c|c|c|c|}
\hline Variável & $\%$ (n) de suspeitos & Valor de $p$ & Odds Ratio & IC $95 \%$ \\
\hline \multicolumn{5}{|l|}{ AME } \\
\hline Sim & $13,33(4)$ & & & \\
\hline Não & $54,54(12)$ & $0,03^{*}$ & 6,93 & $(1,16-41,13)$ \\
\hline \multicolumn{5}{|l|}{ Escolaridade materna } \\
\hline$\leq 8$ anos & $34,28(12)$ & & & \\
\hline$>8$ anos & $23,52(4)$ & 0,533 & 1,70 & $(0,29-9,84)$ \\
\hline \multicolumn{5}{|l|}{ Idade materna } \\
\hline$<18$ anos & $50(1)$ & & & \\
\hline$\geq 18$ anos & $30(15)$ & 0,73 & 0,53 & $(0,01-20,52)$ \\
\hline \multicolumn{5}{|l|}{ Número de filhos } \\
\hline$\leq 2$ & $22,22(10)$ & & & \\
\hline$>2$ & $85,71(6)$ & $0,01^{*}$ & 16,94 & $(1,71-42,87)$ \\
\hline \multicolumn{5}{|l|}{ Peso ao nascimento } \\
\hline$\geq 2500 \mathrm{~g}$ & $33,33(1)$ & & & \\
\hline$<2500 \mathrm{~g}$ & $66,66(2)$ & 0,40 & 4,31 & $(0,13-137,08)$ \\
\hline \multicolumn{5}{|l|}{ Renda familiar mensal } \\
\hline 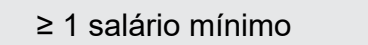 & $22,85(8)$ & & & \\
\hline < 1 salário mínimo & $47,05(8)$ & 0,40 & 0,37 & $(0,03-3,62)$ \\
\hline \multicolumn{5}{|l|}{ Situação conjugal } \\
\hline Vive com companheiro & $26,16(11)$ & & & \\
\hline Vive sem companheiro & $50 \%(10)$ & $0,04^{*}$ & 8,31 & $(1,02-67,24)$ \\
\hline
\end{tabular}

\%: porcentagem; n: número absoluto; IC: intervalo de confiança; AME: aleitamento materno exclusivo até os 6 meses de vida 


\section{DISCUSSÃO}

A caracterização da amostra do atual estudo revelou que as mães e as crianças participantes apresentaram baixo risco biológico, apesar das condições sociodemográficas desfavoráveis. Além disso, a prevalência de aleitamento materno exclusivo (AME) foi superior à média nacional, indicando um percentual de quase $60 \%$ de crianças amamentadas até os 6 meses de vida.

Relatórios do Ministério da Saúde demonstram que o país apresenta uma prevalência de $41 \%$ de AME em crianças de 0 a 6 meses. Apesar do fortalecimento de iniciativas de Promoção, Proteção e Apoio ao Aleitamento Materno e dos evidentes progressos no cenário brasileiro, ainda persistem desafios para se proporcionar condições seguras ao nascimento no país ${ }^{(12)}$. Nos países de baixa e média renda, apenas $37 \%$ das crianças são amamentadas exclusivamente ao seio. Com poucas exceções, a duração da amamentação é menor em países de renda alta do que naqueles carentes de recursos ${ }^{(13)}$.

A amamentação traz inúmeros benefícios para as crianças e para as mulheres e constitui a mais sensível, econômica e eficaz intervenção para a redução da morbi-mortalidade infantil. Sabe-se que os diferentes contextos sociais e culturais podem influenciar a prática do aleitamento materno exclusivo e seus determinantes ${ }^{(14)}$. Os resultados favoráveis apresentados no presente estudo em relação ao índice de amamentação no Brasil podem estar relacionados às ações desenvolvidas pelo Programa Mãe-bebê, que determina como uma das suas atividades prioritárias o estímulo ao aleitamento materno exclusivo através de visitas domiciliares e orientações às gestantes e puérperas em situação de vulnerabilidade social. Seguindo os princípios da Rede Cegonha, o programa fundamenta-se nos princípios da humanização e da assistência, que asseguram às mulheres o direito ao planejamento reprodutivo, à atenção humanizada à gravidez, ao parto e ao puerpério; e às crianças, o direito ao nascimento seguro, ao crescimento e ao desenvolvimento saudáveis ${ }^{(15)}$.

Em relação ao desenvolvimento neuropsicomotor, a amostra estudada na atual pesquisa apresentou uma prevalência de suspeita para atraso no desenvolvimento de $30,8 \%$, similarmente a estudos prévios realizados no Brasil ${ }^{(16,17)}$. Em uma revisão sistemática baseada em investigações brasileiras, os autores observaram que, aproximadamente, $33 \%$ das crianças avaliadas em ambiente domiciliar apresentaram suspeita de atraso no desenvolvimento, sendo que a linguagem foi o domínio mais acometido, seguido da área motora ampla(11).

A primeira infância é uma fase crucial para o desenvolvimento de funções cognitivas e de linguagem. Estima-se que, ao longo dos três primeiros anos de vida, $85 \%$ de todas as conexões neuronais, incluindo as áreas relacionadas à comunicação, são formadas em resposta aos estímulos ambientais. Nos primeiros 12 meses de idade, o domínio da linguagem da TTDD II avalia habilidades que variam desde a emissão de sons e a vocalização de sílabas até a fala de mama/papa específicos. Fatores como baixa condição socioeconômica, idade e escolaridade materna têm sido considerados determinantes para o surgimento de atrasos fonológicos em virtude da diminuição do repertório linguístico, da baixa estimulação da fala e da priorização do cuidado voltado às necessidades básicas da criança ${ }^{(18,19)}$.

Além de déficits de aquisição na linguagem, atrasos no domínio motor-grosso também são referenciados na literatura. As disfunções motoras são os primeiros marcadores observáveis de alterações no desenvolvimento, principalmente em idades mais precoces. Em crianças brasileiras, o aparecimento do controle postural antigravitacional é mais lento quando comparado com crianças canadenses, repercutindo em atraso nas posturas em prono e em pé(20). De acordo com dados da literatura, o progresso no repertório motor amplo é estabelecido, principalmente, em decorrência das atividades que a criança realiza, sendo estas oportunizadas pelo ambiente encorajador e o envolvimento ativo dos cuidadores ${ }^{(21)}$.

O desenvolvimento infantil é multidimensional. Essas dimensões, as quais são interdependentes, incluem desempenho motor, cognitivo, emocional e social, assim como padrões de comportamento, de saúde e status nutricional. Raramente esses aspectos são influenciados de forma individual e isolada. Ao contrário, a combinação de dois ou mais fatores influenciam significativamente na trajetória do desenvolvimento neuropsicomotor ${ }^{(4)}$. Em virtude da característica multifatorial do desenvolvimento, os documentos brasileiros que norteiam a atenção integral na infância recomendam que, para cuidar da criança, educar e promover sua saúde e seu desenvolvimento integral, é importante a parceria entre os pais, a comunidade e os profissionais de saúde, de assistência social e de educação ${ }^{(5,8,22)}$.

A promoção e o acompanhamento do crescimento e desenvolvimento integral da criança formam um dos princípios da PNAISC, priorizando ações de vigilância e estímulo do pleno crescimento e desenvolvimento da criança, em especial do Desenvolvimento na Primeira Infância, pela atenção básica à saúde, conforme as orientações da "Caderneta de Saúde da Criança", incluindo ações de apoio às famílias para o fortalecimento de vínculos familiares ${ }^{(8)}$. 
No presente estudo, a não realização de aleitamento materno exclusivo até os seis meses, a constituição familiar com mais de três filhos e a ausência do companheiro demonstraram forte associação com a suspeita de atraso no desenvolvimento neuropsicomotor nas crianças participantes do Programa Mãe-bebê.

Além de efeitos positivos a curto prazo na saúde da criança, a adequada nutrição e o crescimento saudável trazem benefícios que se prolongam por toda a vida. Em uma coorte de crianças residentes da cidade de Pelotas, Rio Grande do Sul, os autores mostraram uma associação dose-resposta entre a duração do aleitamento materno exclusivo e o aumento da inteligência da criança, do desempenho educacional e da renda aos 30 anos de idade ${ }^{(23)}$. Similarmente ao presente estudo, uma pesquisa realizada no Ambulatório do Hospital Maternolnfantil de Goiânia também evidenciou que a não amamentação exclusiva no seio da mãe até seis meses de vida pode estar relacionada a atrasos no desenvolvimento da criança, principalmente em aspectos como a relação pessoal, social, habilidades manipulativas e grosseiras ${ }^{(24)}$.

Crianças com problemas de desenvolvimento são consideradas mais vulneráveis e requerem suportes específicos, como legislação e políticas públicas de saúde adequadas ao atendimento integral às suas necessidades e à garantia de seus direitos. As principais políticas públicas brasileiras voltadas para a saúde materno-infantil tem como objetivo principal promover e proteger a saúde da criança e o aleitamento materno, mediante a atenção e cuidados integrais e integrados à primeira Infância, do período gestacional aos nove anos de vida, com especial atenção às populações de maior vulnerabilidade, visando à redução da doença (morbidade) e da mortalidade, proporcionando um ambiente facilitador à vida, com condições dignas de existência e pleno desenvolvimento ${ }^{(8)}$.

O leite humano é um fluido complexo que contém lipídeos, proteínas, carboidratos, vitaminas, minerais, substâncias imunocompetentes, além de fatores tróficos ou moduladores de crescimento ${ }^{(25)}$. Para o desenvolvimento do sistema nervoso, o ácido docosahexaenóico (DHA), contido no leite humano, exerce papel fundamental para a maturação neuronal e, consequentemente, para o desenvolvimento de habilidades cognitivas e de linguagem. Em ratos, a deficiência de DHA durante a lactação resultou em pobre retenção da memória durante tarefas de aprendizado ${ }^{(26)}$. Além do aporte nutricional, o aleitamento materno exclusivo também possibilita a construção de um vínculo saudável entre a mãe e o bebê, favorecendo o desenvolvimento emocional, social e psíquico da criança e prevenindo o surgimento de problemas comportamentais em longo prazo ${ }^{(25)}$.

A família desempenha um importante papel de mediadora entre a criança, o ambiente e a sociedade, possibilitando a construção de elementos essenciais para o desenvolvimento infantil e a promoção da saúde e da qualidade de vida. A promoção da saúde, a prevenção de agravos e a detecção precoce de anormalidades físicas e de problemas de desenvolvimento formam o cerne dos programas de vigilância à saúde da criança. Com base nisso, iniciativas globais de promoção à saúde da criança, voltadas à família e à comunidade, têm incentivado práticas de estímulo ao desenvolvimento na primeira infância, a partir de estruturas multissetoriais que incluem: ações de saúde; nutrição; acesso a serviços; ambiente seguro e afetivo; defesa de direitos; proteção e oportunidades de aprendizagem ${ }^{(27)}$.

Além do ambiente familiar, a adequação da estrutura familiar à renda mensal, incluindo o número de filhos, também representa a viabilidade dos recursos básicos para o pleno desenvolvimento de seus membros ${ }^{(28)}$. Núcleos familiares representados por mães que vivem sozinhas com seus filhos indicam um ambiente familiar mais vulnerável, seja no plano econômico, seja do ponto de vista dos cuidados relativos à criança ${ }^{(29)}$.

Sabe-se que a presença do companheiro interfere positivamente na qualidade da estimulação disponível no ambiente familiar ao conferir maior segurança no desempenho da função materna. $O$ fato de as crianças serem cuidadas por pais com união estável e contar com estímulos positivos no lar parece constituir-se em mecanismos protetores para o desenvolvimento no contexto da adversidade psicossocial em que algumas famílias vivem ${ }^{(30)}$. Estudos têm sugerido que o vínculo da criança com o pai é tão importante quanto a relação mãe-bebê. Estima-se que as crianças cujos pais não apoiaram as mães nos cuidados com os filhos possuam, pelo menos, dez vezes mais chance de apresentar suspeita de atraso no desenvolvimento ${ }^{(4,16,31)}$. Os pais, além de ajudarem a prover condições materiais, também ajudam a manter um clima de harmonia e satisfação no ambiente familiar por meio do amor e do companheirismo, promovendo, assim, um ambiente favorável ao desenvolvimento saudável $\mathrm{I}^{(4,16,31)}$.

A interação da criança com os membros da família e com a rede social de proteção assegura não apenas sua sobrevivência, mas contribui para o desenvolvimento neuropsicomotor, cognitivo e emocional. O vínculo da criança com a mãe/cuidador exerce importante papel para a aquisição de habilidades em conjunto com o crescimento e amadurecimento cerebral que acompanham o desenvolvimento. Esse processo é complexo e se apoia na dimensão biológica e psicoafetiva, mas depende estritamente do ambiente para seu florescimento. É na interação com o ambiente familiar, com as redes de cooperação escolar e social mais amplas que a criança poderá se desenvolver plenamente $^{(8)}$. 
Por fim, é importante considerar que, apesar dos avanços, o Brasil ainda apresenta uma imensa desigualdade social, que se reflete diretamente na qualidade de vida das crianças. Dessa forma, reforça-se a necessidade do contínuo investimento em políticas públicas de promoção à saúde e ações para primeira infância e no aprimoramento da qualidade dos serviços e ações já estabelecidos. Cabe lembrar a necessidade da abordagem integral, que não somente garantam a sobrevivência e saúde física, mas que também enfoquem o estabelecimento de vínculo, a promoção de convivência familiar e comunitária saudável, e o desenvolvimento criativo das crianças. Por conseguinte, a articulação de serviços e ações intersetoriais que incluam a área da saúde, educação, desenvolvimento social e assistência, em uma rede solidária para o cuidado integral da criança, são essenciais para atender e monitorar constantemente as famílias em casa e na comunidade, validando a atenção e o suporte, em especial, aos pais de crianças identificadas com atraso ou déficits estabelecidos ${ }^{(32)}$.

A partir dos achados do presente estudo, observa-se que a vigilância do desenvolvimento infantil no primeiro ano de vida deve compreender múltiplas conexões, incluindo não somente os aspectos biológicos, mas também a análise e o acompanhamento de determinantes ambientais, especialmente relacionados à estrutura familiar. Logo, o fortalecimento de políticas públicas brasileiras que valorizem a proteção, a promoção da saúde, a prevenção e a ampliação do cuidado integral à saúde da criança e seu pleno desenvolvimento são fundamentais para a redução dos índices de morbimortalidade infantil. Na perspectiva integral e intersetorial destaca-se, ainda, a necessidade de uma rede de proteção social cuja proposta vise à atenção às necessidades básicas das crianças e de suas famílias, especialmente aquelas em situação de vulnerabilidade social, contemplando ações individuais e coletivas nas áreas de saúde, educação e desenvolvimento social. Dessa forma, as estratégias preventivas e a intervenção precoce voltadas para o neurodesenvolvimento, desde a primeira infância, mudarão o curso de vida dessas crianças e, certamente, refletirão na qualidade de vida da população e em melhores oportunidades socioeducacionais em longo prazo.

O presente estudo apresenta algumas limitações. Em virtude da dificuldade de acesso às residências e à inexistência de dados completos nos prontuários das participantes, a coleta de dados foi realizada apenas em uma parcela da comunidade que participa das ações do Programa Mãe-bebê. Além disso, testes de desenvolvimento infantil validados em crianças brasileiras ainda são escassos. Neste estudo, selecionou-se o TTDDII como instrumento da pesquisa em função da sua ampla normatização e da facilidade de aplicação. No entanto, em virtude de diferenças culturais, os dados podem não refletir o exato desempenho neuropsicomotor das crianças dessa comunidade, uma vez que o teste foi validado em crianças americanas.

\section{CONCLUSÃO}

O presente estudo demostrou que a maioria das crianças $(69,2 \%)$ participantes do Programa Mãe-bebê apresentaram um desenvolvimento neuropsicomotor adequado e compatível com sua faixa etária. No entanto, aproximadamente $30 \%$ das crianças apresentaram neurodesenvolvimento considerado suspeito, sendo os domínios mais afetados a linguagem e o motor grosso. Além disso, observou-se que as crianças participantes do programa que não receberam aleitamento materno exclusivo até os 6 meses e que viviam em famílias com mais de 3 filhos, sem a presença do pai, estiveram em risco para suspeita de atraso no desenvolvimento neuropsicomotor.

\section{AGRADECIMENTOS}

Os autores agradecem à Universidade Feevale pelo apoio para o desenvolvimento desta pesquisa.

\section{CONFLITOS DE INTERESSE}

Não houve conflitos na realização do presente estudo.

\section{CONTRIBUIÇÕES}

Simone de Paula e Ellen Becker Rohr contribuíram com a elaboração e delineamento do estudo; a aquisição, análise e interpretação de dados; a redação e/ou revisão do manuscrito. Maristela Cassia de Oliveira Peixoto, Caroline D’Azevedo Sica e Ilse Maria Kunzler contribuíram com a aquisição, análise e interpretação de dados; a redação e/ou revisão do manuscrito. 


\section{REFERÊNCIAS}

1. Souza JM, Veríssimo MLÓR. Desenvolvimento infantil: análise de um novo conceito. Rev Latinoam Enferm. 2015;23(6):1097-104.

2. Miranda LP, Resegue R, Figueiras ACM. A criança e o adolescente com problemas do desenvolvimento no ambulatório de pediatria. J Pediatr. 2003;79(Suppl 1):S33-42.

3. Ismail FY, Fatemi A, Johnston MV. Cerebral plasticity: windows of opportunity in the developing brain. Eur J Paediatr Neurol. 2017;21(1):23-48.

4. Black MM, Walker SP, Fernald LCH, Andersen CT, DiGirolamo AM, Lu C, et al. Early childhood development coming of age: science through the life course. Lancet. 2017;389(10064):77-90.

5. Ministério da Saúde (BR). Secretaria de Atenção à Saúde. Departamento de Atenção Básica. Saúde da criança: crescimento e desenvolvimento. Brasília: Ministério da Saúde; 2012.

6. Brasil. Ministério da Saúde. Portaria $n^{\circ} 2.446$, de 11 de novembro de 2014. Redefine a Política Nacional de Promoção da Saúde (PNPS). Diário Oficial da União; Brasília, 13 de novembro de 2014.

7. Neves KR, Morais RLS, Teixeira RA, Pinto PAF. Growth and development and their environmental and biological determinants. J Pediatr. 2016;92(3):241-50.

8. Ministério da Saúde (BR). Secretaria de Atenção à Saúde. Departamento de Ações Programáticas Estratégicas. Política Nacional de Atenção Integral à Saúde da Criança: orientações para implementação. Brasília: Ministério da Saúde; 2018.

9. Frankenburg WK, Archer P, Shapiro H, Bresnick B. Denver II: training manual. Denver (USA): Denver Developmental Materials; 1992.

10. Lima SS, Cavalcante LIC, Costa EF. Triagem do desenvolvimento neuropsicomotor de crianças brasileiras: uma revisão sistemática da literatura. Fisioter Pesqui. 2016;23(3):336-42.

11. Brasil. Conselho Nacional de Saúde. Resolução 466/12. Trata de pesquisas em seres humanos e atualiza a resolução 196 [Internet]. Diário Oficial da União; Brasília, 12 de dezembro de 2012 [acesso em 13 Jun 2013]. Disponível em: http://bvsms.saude.gov.br/bvs/saudelegis/cns/2013/res0466_12_12_2012.html

12. Saldan PC, Venancio SI, Saldiva SRDM, Pina JC, Mello DF. Práticas de aleitamento materno de crianças menores de dois anos de idade com base em indicadores da Organização Mundial da Saúde. Rev Nutr. 2015;28(4):409-20.

13. Victora CG, Bahl R, Barros AJ, França GV, Horton S, Krasevec J, et al. Breastfeeding in the 21st century: epidemiology, mechanisms, and lifelong effect. Lancet. 2016;387(10017):475-90.

14. Boccolini CS, Boccolini PMM, Monteiro FR, Venâncio SI, Giugliani ERJ. Tendência de indicadores do aleitamento materno no Brasil em três décadas. Rev Saúde Pública. 2017;51:108.

15. Brasil. Ministério da Saúde. Gabinete do Ministro. Portaria no 1.459, de 24 de junho de 2011. Institui a Rede Cegonha no âmbito do Sistema Único de Saúde - SUS. Diário Oficial da União; Brasília, 27 de junho de 2011.

16. Araujo LB, Mélo TR, Israel VL. Low birth weight, family income and paternal absence as risk factors in neuropsychomotor development. J Hum Growth Dev. 2017;27(3):272-80.

17. Coelho R, Ferreira JP, Sukiennik R, Halpern R. Child development in primary care: a surveillance proposal. J Pediatr. 2016;92(5):505-11.

18. Muluk NB, Bayoglu B, Anlar B. Language development and affecting factors in 3- to 6-year-old children. Eur Arch Oto-rhino-laryngol. 2014;271(5):871-8.

19. Hirsh-Pasek K, Adamson LB, Bakeman R, Owen MT, Golinkoff RM, Pace A, et al. The contribution of early communication quality to low-income children's language success. Psychol Sci. 2015;26(7):1071-83.

20. Saccani $R$, Valentini $N$. Trajetória motora de crianças brasileiras: aquisição do controle postural do nascimento aos 18 meses de idade. Pediatr Mod. 2014;50(8):343-52.

21. Soares ES, Flores FS, Piovesan AC, Corazza ST, Copetti F. Avaliação das affordances presentes em 
diferentes tipos de residências para a promoção do desenvolvimento motor infantil. Temas Desenvolv. 2013;19(106):184-7.

22. Ministério da Saúde (BR). Secretaria de Políticas de Saúde. Área Técnica de Saúde da Criança. Fundamentos técnico-científicos e orientações práticas para o acompanhamento do crescimento e desenvolvimento. Brasília: Ministério da Saúde; 2000.

23. Victora CG, Horta BL, Loret de Mola C, Quevedo L, Pinheiro RT, Gigante DP, et al. Association between breastfeeding and intelligence, educational attainment, and income at 30 years of age: a prospective birth cohort study from Brazil. Lancet Glob Health. 2015;3(4):e199-205.

24. Vieira TS, Amaral M, Fujinaga Cl, Magni C, Mascarenhas LPG. As influências do aleitamento materno para o desenvolvimento infantil: uma revisão integrativa de literatura. Espacios (Caracas). 2017;38(2):1519-30.

25. Girard LC, Doyle O, Tremblay RE. Breastfeeding, cognitive and noncognitive development in early childhood: a population study. Pediatrics. 2017;139(4):1-11.

26. Rathod RS, Khaire AA, Kale AA, Joshi SR. Beneficial effects of omega-3 fatty acids and vitamin B12 supplementation on brain docosahexaenoic acid, brain derived neurotrophic factor, and cognitive performance in the second-generation Wistar rats. Biofactors. 2015;41(4):261-72.

27. Yakuwa MS, Neill S, Mello DFd. Estratégias de enfermeiros para a vigilância à saúde da criança. Rev Latinoam Enferm. 2018;26:e3007.

28. Santos JC, Freitas PM. Planejamento familiar na perspectiva do desenvolvimento. Ciênc Saúde Colet. 2011;16(3):1813-20.

29. Melo SC, Marin AH. Influência das composições familiares monoparentais no desenvolvimento da criança: revisão de literatura. Rev SPAGESP. 2016;17(1):4-13.

30. Defilipo ÉC, Frônio JS, Teixeira MTB, Leite ICG, Bastos RR, Vieira MT, et al. Oportunidades do ambiente domiciliar para o desenvolvimento motor. Rev Saúde Pública. 2012;46(4):633-41.

31. Sethna V, Perry E, Domoney J, lles J, Psychogiou L, Rowbotham NEL, et al. Father-Child interactions at 3 months and 24 months: contributions to children's cognitive development at 24 months. Infant Ment Health J. 2017;38(3):378-90.

32. Lari LV, Lourenço GF, Barba PCDSD. Legislações e documentos brasileiros sobre a atenção à criança e suas implicações para o monitoramento do desenvolvimento infantil. Da Investigação às Prat. 2018;8(2):4-20.

\author{
Autor para correspondência: \\ Simone de Paula \\ Universidade Feevale \\ ERS-239, 2755 \\ Bairro: Vila Nova \\ CEP: 93525-075 - Novo Hamburgo - RS - Brasil \\ E-mail: sdpaula@feevale.br
}

Como citar: Paula S, Rohr EB, Peixoto MCO, Sica CD, Kunzler IM. Análise do desenvolvimento neuropsicomotor de crianças participantes de um programa mãe-bebê. Rev Bras Promoç Saúde. 2019;32:8603. 